\title{
Methodologies for Passive Seismic Event Location: based on Wave Propagation
}

\author{
Bulcão, A.; Alves, G.C.; Dias, B.P.; Soares Filho, D.M.; Cardoso da Silva, A.A.;
}

Copyright 2019, SBGf - Sociedade Brasileira de Geofísica

This paper was prepared for presentation during the $16^{\text {th }}$ International Congress of the Brazilian Geophysical Society held in Rio de Janeiro, Brazil, 19-22 August 2019.

Contents of this paper were reviewed by the Technical Committee of the $16^{\text {th }}$ International Congress of the Brazilian Geophysical Society and do not necessarily represent any position of the SBGf, its officers or members. Electronic reproduction or of any part of this paper for commercial purposes without the written consent of the Brazilian Geophysical Society is prohibited.

\section{Abstract}

The knowledge of passive seismic events source location in subsurface is important for many different applications in Geophysics and Engineering, such as Reservoir Monitoring, location of earthquake hypocenters and micro-seismic events, risk mitigation during well drilling due to fracture zones, local stress orientation for Geomechanics analysis, etc...

In this paper, we present and test two distinct methodologies based on wave propagation to search the source location of passive seismic events. The first one takes into account the wave equation temporal reversibility, where the location for the passive source is stablished as the point where the energy is concentrated as we back-propagate the recorded passive event. The second one uses the objective function normally employed by Full Waveform Inversion (FWI) schemes, where observed and calculated seismic datasets acquired at the receivers' location are compared via an objective function. However, unlike traditional FWI schemes, instead of updating the velocity model, the optimization searches for the source location through a global optimization scheme.

We apply both methodologies in a dataset acquired in a Brazilian oil field using an Ocean Bottom Cable (OBC) seismic survey. We employ the primary and the firstorder surface-related multiple events to try to enhance the location's resolution.

\section{Introduction}

The dataset employed in this work comes from a PRM (Permanent Reservoir Monitoring) system that was deployed in 2012 at the Jubarte oil field (that belongs to Petrobras and is located in Brazil's Campos basin). It was the first of its kind, with the goal to serve as a pilot test of a fully fiber-optic system deployed at 1300 meters water depth, with 712 four components (4C) receivers covering a very small area of approximately $9 \mathrm{~km}^{2}$. Figure 1 shows a draft of the survey geometry. It was active during several air gun campaigns and also used for passive monitoring (Thedy et.al, 2015 and Goertz et.al, 2015).

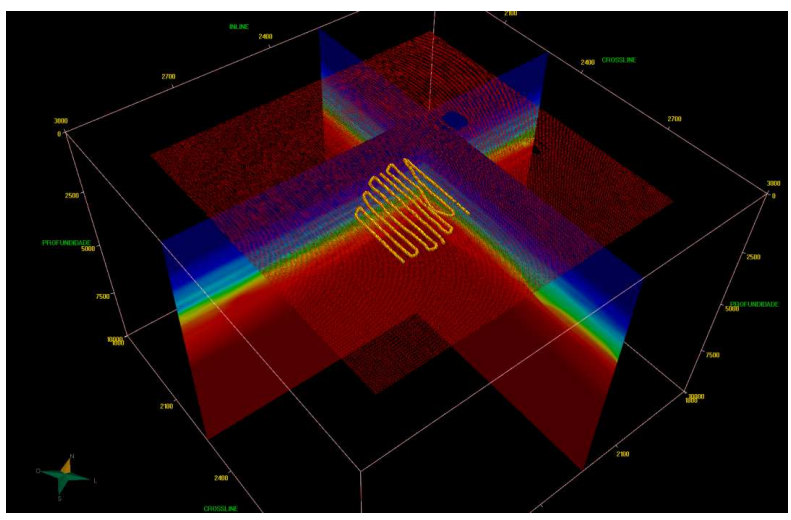

Figure 1 - Schematic scheme of Jubarte PRM system, where the yellow line represents the deployed OBC cables (cover around $9 \mathrm{Km} 2)$, the red dots at surface are the shot pattern $(25$ by 25 meters, in-line and cross-line respectively) and the two orthogonal planes display the migration velocity model (with values from 1495 to $5580 \mathrm{~m} / \mathrm{s}$ ).

During the passive seismic campaign, micro-seismic events, possibly of natural origin, were recorded with moment magnitudes ranging from 0.2 to 1.9. Most of them were detected with a couple hours window interval and with an estimated depth of about $5 \mathrm{~km}$ near an area of a developed reservoir (Goertz et.al, 2015).

\section{Dataset Employed}

Previous works done by service companies PGS and MicroSeismic, INC (Goertz et.al, 2015) analyzed the entire passive dataset. In this paper, we selected a specific passive seismic event. We localized this event in alignment with one of the OBC cables, making it suitable for a 2D study. Figure 2 illustrates a map view zoom of the passive source location (black mark, defined previously), the 2D selected velocity model (green line) and the receivers' locations (red dots).

Using the available 3D migration velocity model, we extracted a 2D line and interpolated the grid spacing to a 10 meters interval. Figure 3 shows the 2D velocity model.

The pre-processing workflow that was originally applied to the dataset consists of a band pass filter $(4-8-35-40 \mathrm{~Hz})$, rotation of the 3 components' data to enhance the recorded compressional direct wave and polarity check after the 3D rotation. 


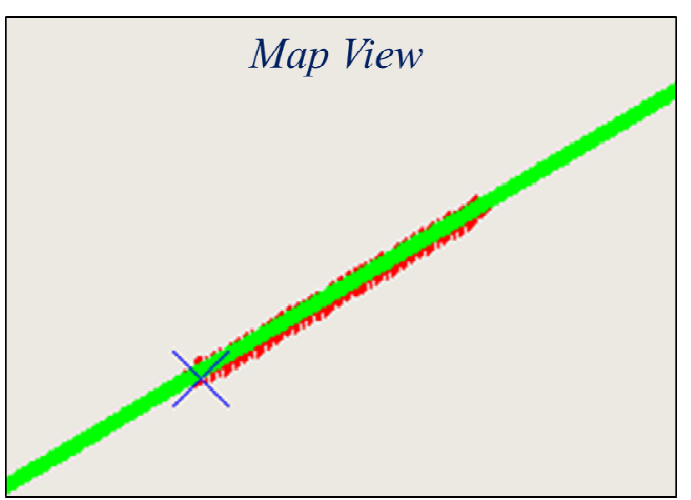

Figure 2 - Schematic map view zoom, displaying the passive source location (black mark, defined by the services companies), the 2D line selected contained the velocity model (green line) and the receivers' locations (red dots) throughout the OBC cable.

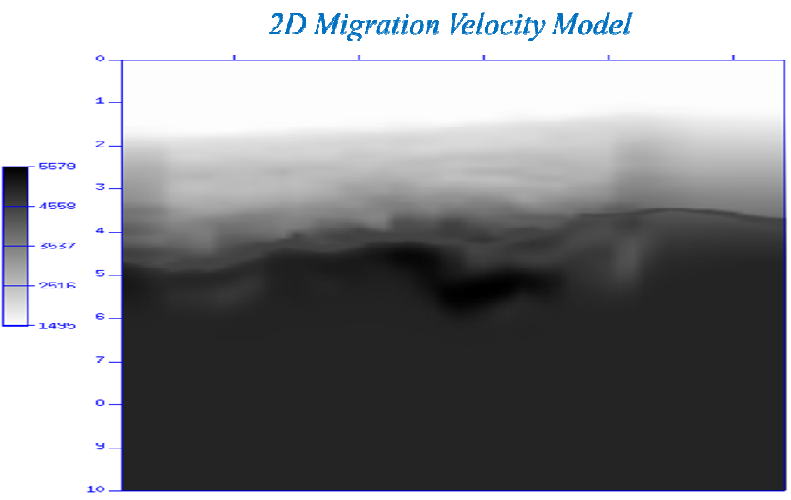

Figure $3-2 D$ velocity model, extracted from the 3D depth migration velocity model. It was used during the backpropagations and all the numerical simulations done along in this work.

Figure 4 shows the selected passive seismic event windowed around the primary and first-order surfacerelated multiple (as indicated in the picture). Observe that we established the time origin in an arbitrary position, as the passive event starting time is one of the unknowns. We also assume that the event has an impulsive shape, so that the first arrival represents it correctly.

\section{Methodologies and Application}

Both methodologies we present assume that the properties models employed for the wavefield reconstruction are sufficiently accurate and are a good representation of the real model. Usually, a migration velocity model will be adequate for this propose.

In this work, we use a simple isotropic acoustic wave propagation model to simulate the wavefield. We emphasize that a more realistic and elaborated mathematical model could be employed without changing any of the procedures presented in this work, for instance, utilizing models that include anisotropy and/or elastic mode conversions.

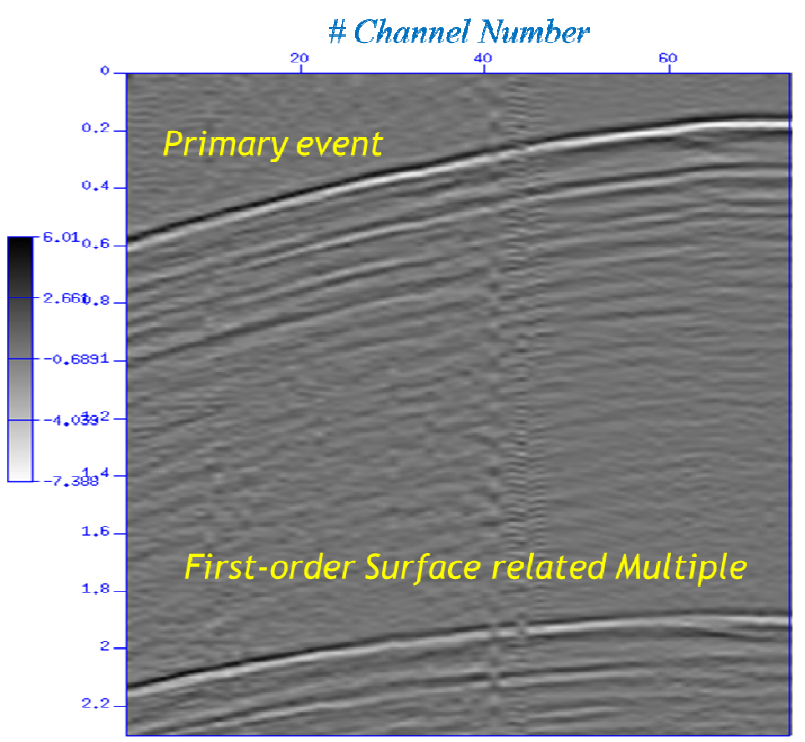

Figure 4 - Passive seismic event selected to test the presented methodologies. In the picture is indicated the primary and first-order surface-related multiple events.

We explore two distinct and complementary methodologies in this section, which can be used consecutively as a way to enhance the source location resolution. It is also possible to apply both of them to different acquired data, like primary events only, surfacerelated multiples, or the entire dataset (primaries + multiples).

The first method we present is based on the wave propagation temporal reversibility principle. Using this principle, the observed passive seismic event (seismogram) is back-propagated through the velocity model, by re-injecting the data at the receivers locations. According to this principle, the energy will be travelling backwards in time and will focus at the source location.

As data are continuously recorded in passive seismic acquisitions, the initial time for a passive seismic event is unknown. For this reason, we apply a time window around the identified seismic event, which we backpropagate and then evaluated the simulated wavefield at some specific time steps, looking for the absolute maximum value (greatest localized energy) and its location (we used every 0.06667 seconds).

To clarify this, figure 5 illustrates our workflow for the primary event only. It is possible to see the snapshots for the wavefield being back-propagated considering different time steps. Following our procedure, the snapshot that presented the largest absolute maximum value was the one labeled as time step 1600 (arbitrary counter), with grid point coordinates at $X=391801, Y=7646680$ and depth 4920 meters (already in the global reference system). 


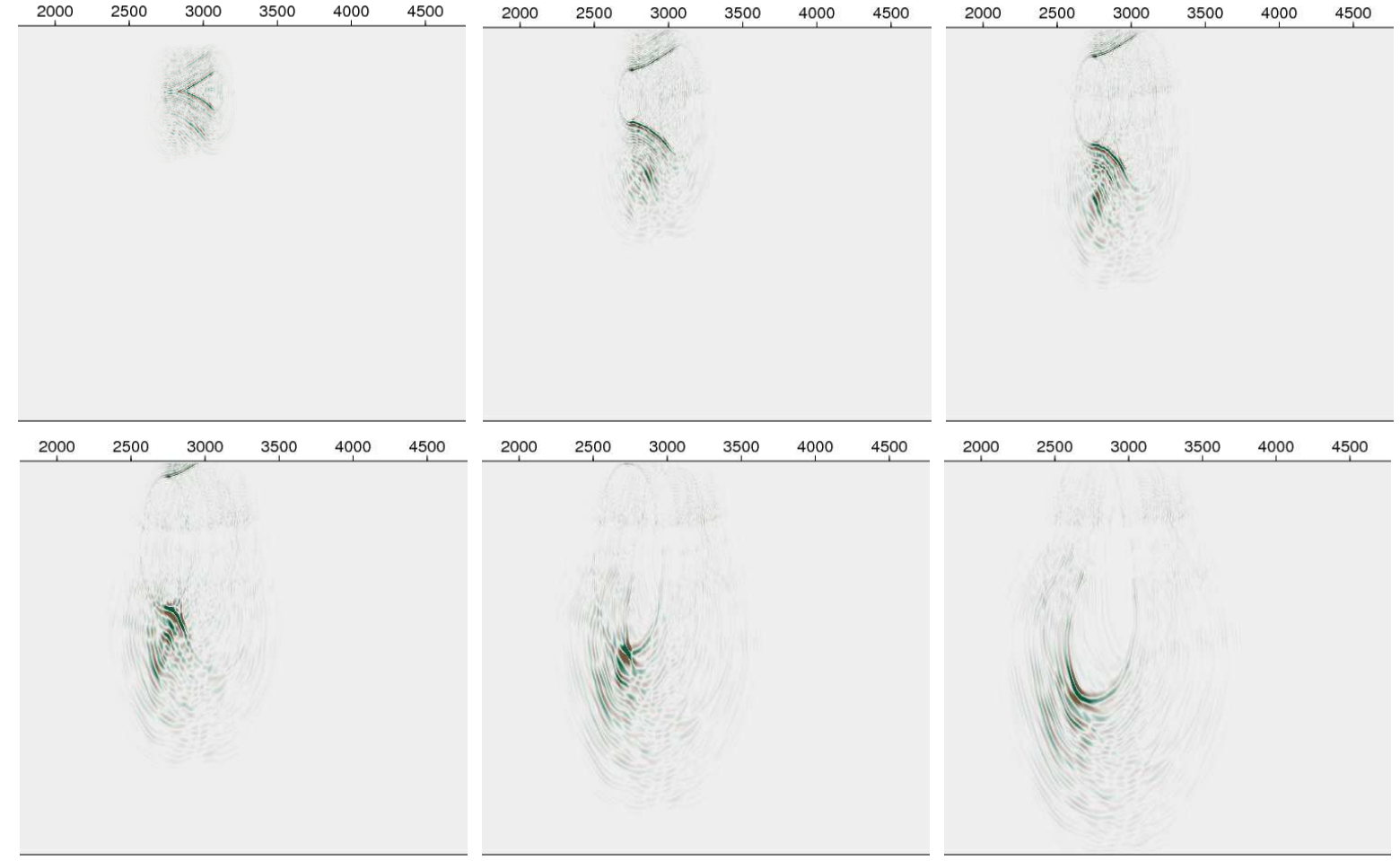

Figure 5 - Some selected snapshots during the back-propagation process. The arbitrary number that represents the propagation time starts backwards from 3600 to 1200 with a 400 time steps spacing, from left to right and top to bottom. The energy focusing could be observed at the penultimate picture.

Afterwards, we employed the second procedure as a way to enhance the source location of the passive event. This procedure is based on the evaluation of the traditional objective functional usually employed in FWI. In this case, we adopt the $\mathrm{L} 2$ norm between the difference of the observed (real seismogram) and calculated (numerically simulated) datasets, with a simple Ricker wavelet as the source in all numerical simulations. We use the first arrival at the receivers' positions to match the time between datasets, as the time origin for passive events in observed data is arbitrary.

To evaluate the sub-space for the optimum solution, i.e. different passive source locations, we adopt a basic global optimization search engine, in which a regular grid is defined around the first guess, considering a 5 meters interval and offsets of 40 meters inline and 60 meters in depth. Figure 6 represents the objective functional. We interpolate bi-linearly between evaluated points to produce a smoother solution surface.

In figure 6 , the lowest value for the objective functional corresponds to the point with coordinates $X=391828.08$; $Y=7646653.33$ and 5125 meters depth. This point was selected as the enhanced passive source location.

We can also demonstrate that the same procedures can basically be applied independently for first-order surface related multiples and/or for the entire dataset composed by primaries and multiples events. To achieve that goal, it is necessary to apply a slight modification on the velocity model, by extending the water layer artificially to mirror the receivers' positions over the sea surface (similar to the mirror migration procedure, Bulcão, 2004 and Zhang et.al 2013). Figure 7 illustrates this idea schematically.

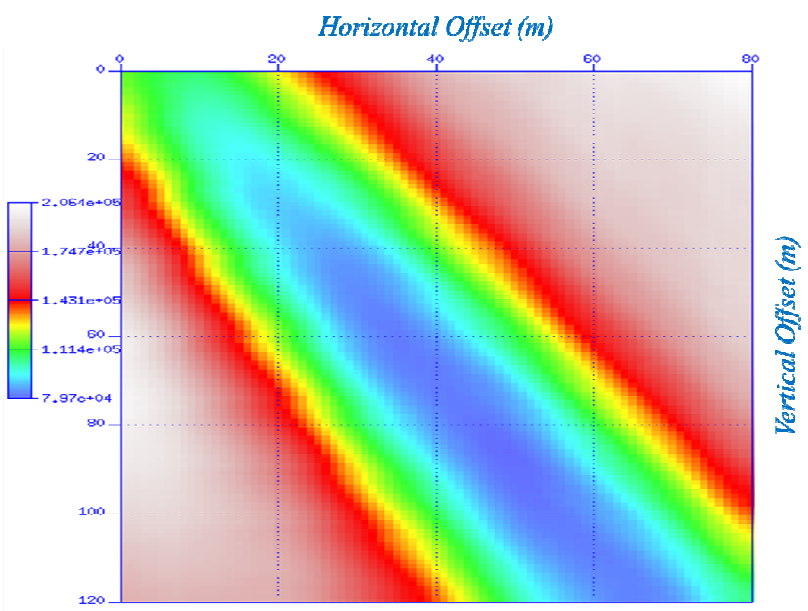

Figure 6 - L2 Norm objective functional Illustration, considering different possible sources positions for the passive seismic event. The values were interpolate bi-linearly between evaluated points to produce a smoother solution surface. The minimum (lowest value) represents the optimized source location. 


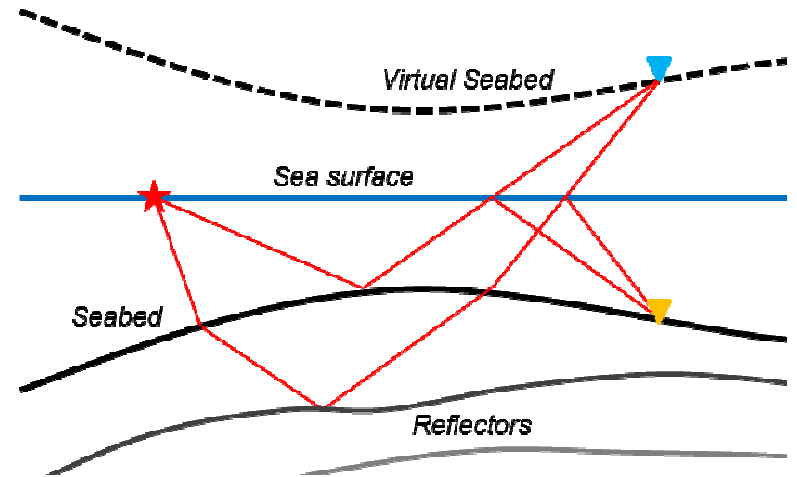

Figure 7 - Sketch for modifying the model properties to consider the virtual receivers (mirroring the actual receivers' positions over the sea surface, yellow triangle), in order to injecting the first-order surface related multiple event at the virtual receivers (cyan triangle).

Next, we back-propagate the passive data windowed around the first-order surface related multiple associated to the event. We start by prescribing it at mirror positions to the receivers' and then evaluate the absolute maximum value for different snapshots through the numerical simulation at different time steps. The new estimation for the source location was found at $X=391801 ; Y=7646680$ and 4910 meters depth.

Using both primary and first-order surface related multiples simultaneously for the back-propagation, the source location found was $X=391801 ; Y=7646680$ and 4920 meters depth.

Table 1 summarizes results obtained for the first source location methodology considering different datasets: primaries only; first order multiples; and both together. One interesting aspect that arises from these results is the confirmation of the superposition principle, as the amplitude for both events together should match the sum of then independently. We obtain this result with only a difference of 0.38 percent.

Table 1 - Results for the first Methodology applyed

\begin{tabular}{|l|l|l|l|}
\hline Event Type & $\begin{array}{l}\text { Trace } \\
\text { Number }\end{array}$ & Depth & $\begin{array}{l}\text { Maximum } \\
\text { Amplitude }\end{array}$ \\
\hline Primary Only & 2665 & 4920 & 5.156 \\
\hline Multiple Only & 2665 & 4910 & 3.377 \\
\hline Primary + Multiple & 2665 & 4920 & 8.501 \\
\hline
\end{tabular}

\section{Conclusions}

The results we obtained and analyzed in this work, together with some previous exercises done by PGS and Microseismic Inc. (Goertz et.al, 2015), allow us to conclude that:

Each methodology we present has different characteristics regarding the domain where data is compared/evaluated: (i) In the back-propagation scheme, we compare time slices of the wavefield, i.e., in space domain (similar to the velocity model).

(ii) In the objective functional scheme, we compare the seismograms acquired and modeled, i.e., in the data domain.

We demonstrate that the use of surface related multiples can be employed as an additional information beyond the primaries, as to improve the source location accuracy.

Both methodologies we present reached promising results. The difference between the passive seismic source location from the service company and those we calculated was less than 125 meters in a depth around 5000 meters.

Part of the difference could be due to 2D limitations in our application. In this work, the main goal was to evaluate applicability of both methodologies. In the future, we plan to extend the results and applications to 3D.

\section{Acknowledgments}

The authors would like to thanks Petrobras to authorize the presentation of this work and specially the geophysicists Ruan Giacomini Couto, Wilson Lisboa Ramos Filho e Wendel Lopes Moreira for their support with the data acquisition and original processing, data gathering and information about the field development history.

\section{References}

Bulcão, A.; 2004; Migração Reversa no Tempo de Dados Sísmicos Empregando Operadores Elásticos, Tese de Doutorado (in Portuguese), UFRJ/COPPE.

Goertz, A.V.; Richardson, J.; Faragher, J.; Remington, C.; Morton, P.; Barros, P.; Theodoro, C.E.; 2015; Microseismicity Recorded by a Fiberoptic Oceanbottom PRM Installation Offshore Brazil; Third EAGE Workshop on Permanent Reservoir Monitoring.

Thedy, E.A.; Dariva, P.; Ramos Filho, W.L.; Maciel Jr, P.O.; Silva, F.E.F.; Zorzanelli, I.B.; 2015; First Results on Reservoir Monitoring in Jubarte PRM Offshore Brazil; Third EAGE Workshop on Permanent Reservoir Monitoring.

Xuan, R.; Sava. P.; 2010; Probabilistic microearthquake location for reservoir monitoring, GEOPHYSICS.

Zhang, D., and G. Schuster, 2013, Least-squares reverse-time migration of multiples: Geophysics 